\title{
PRESENTACIÓN DEL N 5 AÑO 6 (2010) DE LA REVISTA DE LA ESCUELA DE CIENCIAS DE LA EDUCACIÓN, UNR
}

\author{
Carolina Kaufmann \\ (Universidad Nacional de Rosario. Universidad Nacional de Entre Ríos)* \\ ckaufmann@express.com.ar
}

A continuación se presentan las palabras brindadas por la Dra. Carolina Kaufmann en la presentación del № 5 Año 6 (2010) de la Revista, el día 7 de junio de 2011. A los efectos de comprimir las informaciones brindadas en la exposición oral, se ha reducido en esta versión escrita algunas gráficas presentadas oportunamente ( 5 en total) y que daban cuenta del crecimiento exponencial, del ritmo y niveles del volumen de páginas y del incremento de las variables presentadas en los diferentes números publicados.

\section{Lo transitado}

Saludo a colegas, jóvenes colegas, estudiantes e invitados que hoy nos acompañan. Agradezco la invitación a participar en este Panel y me disculpo públicamente por las invitaciones anteriores que no pude concretar.

Mi participación en este Panel de Presentación del 5to número de la Revista de Ciencias de la Educación pivoteará sobre tres puntos: Lo transitado, La cocina de la Revista y Pensando en mañana. Mi intervención inicialmente sesgada por mi oficio, no puede soslayar un breve balance y prospectivas del camino recorrido realizado hasta la actualidad por la Revista. En principio efectuaré una mirada de conjunto en el discurrir de la Revista que hoy nos convoca.

Comienzo centrando la lente en sus primeros pasos, y según se definían como objetivos explícitos en el año 2005:

Construir un instrumento de divulgación y difusión de producciones académicas y/o de investigaciones realizadas por los docentes, in-

\footnotetext{
* Doctora en Educación por la Universidad de Valladolid, España. Magíster en Educación con Orientación en Historia y Prospectiva (UNER). Licenciada y Profesora en Filosofía (UNR). Profesora Titular regular de Historia Social de la Educación en la Facultad de Ciencias de la Educación (UNER) y Profesora Titular regular del Núcleo Histórico-Epistemológico de la Educación en la Facultad de Humanidades y Artes (UNR). Dirige el Grupo TIPHREA-UNER (Tendencias ideológico/pedagógicas en la historia reciente de la educación argentina).
} 
vestigadores y alumnos (con aval docente) de la carrera de Ciencias de la Educación y/o de otros docentes e investigadores de nuestra Facultad y otras facultades de la UNR, de otras Universidades Nacionales e Internacionales comprometidas con las problemáticas educativas.

Desde estas expectativas institucionales, y durante los dos primeros años de su publicación, los objetivos se tradujeron en perfiles y algunos indicadores bibliométricos que se plasmaron en Tabla 1 en la que puede apreciarse la significativa evolución de la Revista en sus años de existencia.

Sin duda, el crecimiento del número de contribuciones fue incrementándose con los años, llegando a un nivel óptimo de publicaciones sin declinar, lo cual continúa marcando el perfil de la Revista. Información sobre las instituciones de pertenencia de los autores se puede encontrar en la Tabla 2.

El aumento del volumen de páginas, también incluyó la apertura a un mayor abanico de Áreas temáticas tales como formación docente y universidad; los problemas del lenguaje; los aportes sobre las nuevas tecnologías; el aprendizaje atravesado por los diagnósticos y sus efectivas posibilidades; la enseñanza de las ciencias; adultos mayores y educación intergeneracional; historia de la educación: nuevos relatos para reconocidas experiencias; democratización del conocimiento, escuela secundaria, aprendizajes en movimiento y estudios de casos, marcan un abanico de problemas significativos que apuntan a no desestimar el amplio espectro, en la medida en que se incluyen inquietudes y parcelas cognitivas estudiadas en la actualidad por las ciencias de la educación.

\section{La cocina de la revista}

En los inicios se observaba claramente que el orden que la Revista debía priorizar a la:

(...) producción y difusión de temas teóricos o aplicados, que involucren a los cuerpos académicos de nuestra institución e instituciones académicas locales, regionales e internacionales, comprometidos con campos de conocimientos vinculados a diferentes temáticas educativas (p. 1 Reglamento).

En este sentido, no quedan dudas del crecimiento cuantitativo de la Revista, en tanto que, las Normas para publicación, ilustran los debates -no concluidos- en el seno de los miembros del Comité Editorial, así como también un perfil que va contorneándose más nítidamente número a número. Por caso, en las Normas del Año 2, № 1, sólo se solicitaba que "Los trabajos presentados deberán ser inéditos, y no estar en proceso de evaluación en ninguna otra publicación "(p. 253). Años más tarde, se establecerán las distintas Secciones que conformarán la revista: 
- Artículos científico-académicos con referato.

- Artículos libres sin referato, cuya publicación es orientada por el Comité

- Editorial.

- Trabajos de alumnos de la carrera de Ciencias de la educación, avalados por docentes de la carrera (requisito necesario para su aceptación al momento de la entrega del mismo, mediante una nota escrita y firmada).

- Reseñas de tesinas/tesis finalizadas, defendidas y acreditadas.

- Reseña de libros y eventos.

Precisamente, en este número que hoy presentamos, las Secciones que conforman la Revista, adquieren una nueva normativa y requerimientos cualitativos más cercanos a propuestas editoriales con mayor nivel de exigencias y/o excelencias científicas:

- Artículos científico-académicos con Referato.

- Trabajos de alumnos/as de la carrera de Ciencias de la Educación, avalados fehacientemente por comunicación escrita y firmada por docentes de la carrera, requisito necesario para su aceptación al momento de la entrega con referato.

- Reseña de tesinas con defensa concluida y avaladas por sus Directores/ as, con idéntico requisito al punto 3 con referato.

- Reseña de libros con referato.

- Reseña de eventos con referato.

Indudablemente, se observa una progresiva amplitud de contribuciones por fuera del marco de la propia Escuela de Ciencias de la Educación y de instituciones universitarias argentinas. En esta oportunidad, se cuenta con tres trabajos de universidades extranjeras: Colombia, Brasil y México.

Ahora bien, quienes nos sentimos comprometidos con este espacio -de distintas maneras- respecto de su continuidad y consolidación, esta etapa presenta varios desafíos que se vienen debatiendo en el seno de las reuniones del Comité Editorial. Precisamente, este número dedicado en memoria del Prof. Edgardo Ossanna, remite a sus propias palabras escritas en un Informe a los miembros del Comité Académico Interno hace un año atrás, el día 20 de junio de 2010 en ocasión de solicitar a todos los miembros del Comité Editorial nuestros comentarios y aportes para enriquecer la revista. Por entonces, Edgardo explicitaba "sin ánimo de ser esquemático" y en función de su organización y diagramación, su preocupación "acerca de qué se pretende con la revista: si es una revista científica o es una revista de circulación". Agregaba:

Si la definición es mixta debe quedar bien claro en la organización de la revista, cuál es la parte central, la de los trabajos científicos, y cuál la que corresponde a trabajos en preparación, primeros aportes, posibilidades de los que se inician. 
Comparto sus inquietudes y considero que merecen ser retomadas y analizadas a los efectos de lograr un mayor nivel de definición o de opción; esto es: si se decide sostener la priorización marcada por los objetivos primigenios o si los mismos deben revisarse y actualizarse. En síntesis, sus palabras aún resultan centrales.

Por otro lado, desde una perspectiva general y sin pretender ceñirnos a reduccionismos, observando toda la producción del número que hoy se presenta, se ofrecen trabajos sobre una diversidad temática que han superado las instancias de un doble referato que nos exime de realizar ponderaciones sobre los mismos. Señalamos de modo ilustrativo tres grandes ejes orientadores que caracterizan este número de publicación:

- Teórico-histórico-comparada que agrupa trabajos centrados en torno a distintos aspectos de la educación superior, secundaria.

- Tecnológica (práctica) que acumula movimiento.

- Estudios de casos.

El primer bloque recoge problemáticas cuyas finalidades resultan eminentemente reflexivas y analíticas vistas en diferentes perspectivas. El segundo tercer bloque incluye trabajos de corte más pragmático sin que esta invalide el recurrir a esquemas de te fundamentación metodológica. En ese sentido, este número se nutre de:

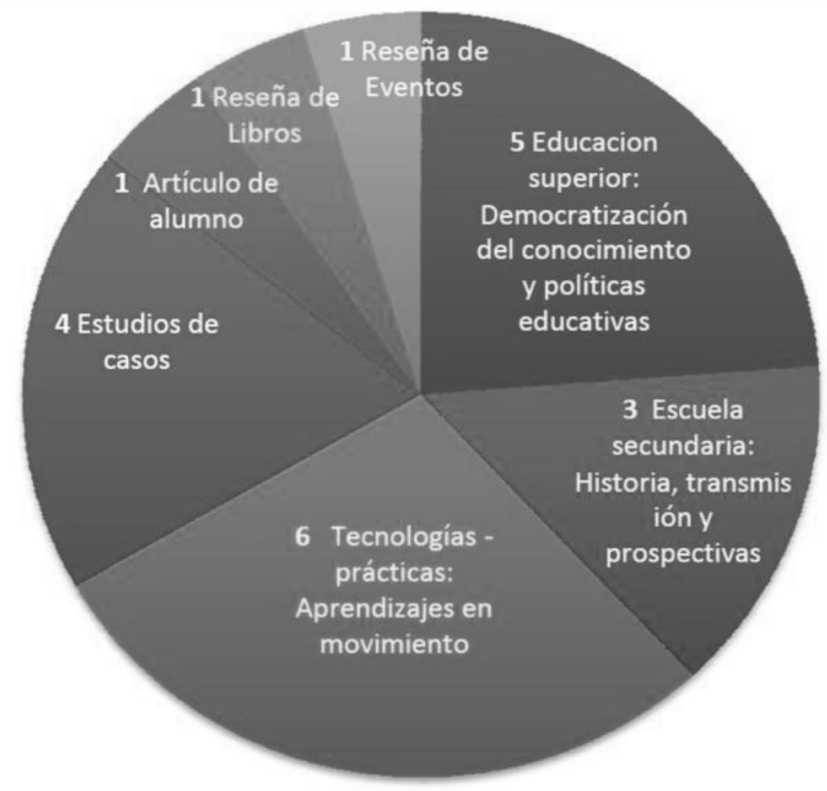

Figura 1. Distribución de las contribuciones publicadas en el año 2010 
Específicamente:

\section{A. Educación Superior: Democratización del conocimiento y políticas educativas en educación superior}

- Observaciones a la actual configuración del proceso de trabajo docente en la educación superior. Aportes desde la experiencia sindical y la producción de cátedra, a las nociones de subjetividad y trabajo docente en la educación superior. La cultura académica tras la agenda del mercado, Gustavo Brufman.

- La retórica institucional del banco mundial en torno a las políticas de ciencia y tecnología de las universidades latinoamericanas. Hacia la búsqueda de autonomía académica en los países de la región, Mariano Diodati, María Graciela Mendíaz.

- Educación a distancia y formación docente. Retos y desafíos en los postgrados de la Universidad Nacional de Rosario, Susana Copertari, Natalia Sgreccia, Yanina Fantasía.

- Extensão universitária: a aposta pela democratização do conhecimento, Leao Alcides.

- La carrera docente en la Universidad Nacional de Rosario, Rosanna Schanzer, Ma. Cecilia Muruaga.

\section{B. Escuela Secundaria: Historia, transmisión y perspectivas}

- La evolución del régimen de disciplina en el nivel medio de enseñanza. Ciudad de Buenos Aires, Argentina (1943-2010), Natalia Álvarez Prieto.

- Transmitir el pasado reciente... una reflexión sobre los actos del día de la memoria por la verdad y la justicia en las escuelas medias de Rosario, Paula Caldo, Elvira Scalona.

- ¿Qué profesores necesita esta escuela? Notas en torno a la formación docente universitaria de cara a las exigencias que plantea una escuela secundaria para todas/os. Noelia Andrea Orienti.

\section{Aprendizajes en movimiento (TECNOLÓGICA/PRÁCTICA)}

- La configuración de la identidad de los sectores populares en la era de la globalización. Apuntes para pensar las prácticas escolares, Natalia Jorgelina Forlini.

- El revés de la trama: elegir una carrera/recibir estudiantes en una carrera, Analía Umpierrez.

- La construcción de lo público mediado por un dispositivo hipermedial dinámico, Patricia S. San Martín, Silvana Martino.

- Errores de los estudiantes de octavo grado en el trabajo pre-algebraico, Tulio Rafael Amaya de Armas. 
- $\quad$ El concepto de variación: sus inicios en la educación básica y repercusión en la educación superior, Elena Fabiola Ruiz Ledesma.

- La institución escolar: ¿encuadre o desacople en la formación permanente docente? Una aproximación a las concepciones docentes, Irene Macera.

\section{Estudios de casos}

- Los textos escolares para primer año en el ciclo básico unificado. Estudio de casos en río negro, período 1989-1995, Nancy Liliana Salerno.

- La Unco y el desarrollo de grupos académicos. El caso del "ecotono" en su etapa fundacional, María de Los Ángeles Colodro.

- La comunicación en la virtualización de la educación. El caso del campus virtual Puntoedu, María Cristina Alberdi.

- Aproximaciones a la subjetivación sexo-genérica en la profesión docente. Estudio comparativo de tres cursos de formación docente en educación sexual en Córdoba, Tomás losa.

\section{E. Artículos de alumnos avalados con referato}

- El proceso de construcción del nuevo diseño curricular para el profesorado de educación primaria $n^{\circ} 528 / 09$ en la provincia de Santa Fe, Mauro Carbonari, Camila Carlachiani, Romina Craparo.

\section{F. Reseña de libros con Referato}

- Dejar caer el agua, Natalia García.

\section{G. Reseña de eventos}

- III Jornadas de Formación Docente Universitaria: Debates contemporáneos y nuevos escenarios para la formación docente universitaria, Silvia Morelli

\section{Pensando en mañana}

Deseo subrayar, ya que me consta, el enorme esfuerzo del Comité Editorial coordinado por la Profesora Susana Copertari, así como también el sistemático trabajo realizado por los jóvenes alumnos y docentes involucrados en la concreción de la Revista, su rigurosidad y minuciosidad en las tareas asumidas. Desde los requisitos formales que involucran una publicación de este tipo en el que se comenzó a transitar por caminos no recorridos previamente que involucraron las actividades de digitalización, la preocupación por regirse según los criterios homogéneos y explícitos de calidad editorial hasta las dificultades por contar con los recursos económicos suficientes y la genuina preocupación por ampliar su circuito de circulación que posibilite lograr mayor visibilidad e 
intercambio institucional con múltiples ámbitos académicos. Y "como si esto fuera poco", dicho en el más estricto sentido de los pregones de vendedores callejeros-, decimos que todo este esfuerzo editorial y hasta el día de la fechaestá cimentado en el trabajo ad-honorem del equipo editorial y cero registro/ reconocimiento institucional (concretamente en una resolución que acredite las funciones de los responsables de la Revista). Hasta aquí, todo ello, es parte de la "cocina de la Revista".

Cocina invisible para quienes no participan de este circuito de producción editorial. Recordamos que ya en la anterior presentación del número 4, año 5 , 2009, la Dra. María Teresa Pérez Lariño, mencionaba:

(...) la necesidad de incluir la Revista en la Base de Datos Bibliométricos", y además "valorar la edición electrónica de la revista. Una salida en Internet, teniendo en cuenta el movimiento de Acceso Abierto (Open Access) que promueve el acceso a la información científica" que permitiría ampliar la visibilidad de la revista al permitirle llegar a un público mayor que el disponible mediante la circulación de una cantidad limitada de números.

Esta es una deuda pendiente que pronto habrá de saldarse. Al respecto, en la presentación de este número, la directora señala que "...es necesario aclarar que estamos en proceso de tramitación para la inclusión de la Revista al "Sistema de Indexación del Latindex". Ello implicó, previamente, un proceso de debate interno en el que no estuvieron ausentes las distintas voces que componen un largo debate académico al interior del Comité Editorial y del Comité Académico Interno de la Revista junto a la Dirección de la misma. Los lectores, tendrán ahora la palabra para ponderar los aportes de los trabajos presentados en este número, tarea que nos supera en esta presentación.

Mi recorrido por el trayecto de la revista y a la luz de lo argumentado, evidencia que esta publicación desde sus inicios ha seguido una política editorial persistente y definida con posibilidades de crecimiento observada en múltiples indicadores: no sólo lograr la periodicidad en sus publicaciones, la rigurosidad de los mecanismos de arbitraje sino también el avance en el nivel de exigencias y consecuente excelencia en sus resultados. Este proyecto editorial nacido de las necesidades propias de la escuela de Ciencias de la Educación, no puede omitir que ha sido y es un proyecto democrático con niveles de consulta y participación amplia a los miembros de los distintos estamentos de la Escuela. Las reuniones abiertas semanales del Comité Editorial, las permanentes consultas al Comité Académico Interno, las sucesivas Actas, los Informes de Gestión de lo realizado y los números ya publicados dan cuenta de estas afirmaciones. También deseo destacar el invalorable apoyo que nos brinda permanentemente nuestro editor Poly Laborde a quien le estamos muy agradecidos. Celebro lo mucho que se ha hecho en estos seis años de existencia de la Revista. 
Aspiramos entonces que igual empuje y efectivizarían de resultados se multipliquen en otros ámbitos de la carrera que se estiman demorados; fundamentalmente en lo referido a la revisión y ajuste del Plan de Estudios puesto en marcha en 1985 y del que no me siento ajena. Si demandamos rigurosidad y calidad en las producciones y publicaciones, ello no puede estar desarticulado de iguales exigencias, reales y formales, en un Plan de carrera forjado por actores y para actores del campo educativo. A once años de iniciado el siglo XXI, ciertos cambios ya no admiten dilaciones. ¿Caben dudas de la capacidad institucional propia y del conjunto de sus actores para lograrlo? Este recorrido pincelado por el devenir de la Revista, constituye una apretada muestra de las potencialidades aún latentes. 


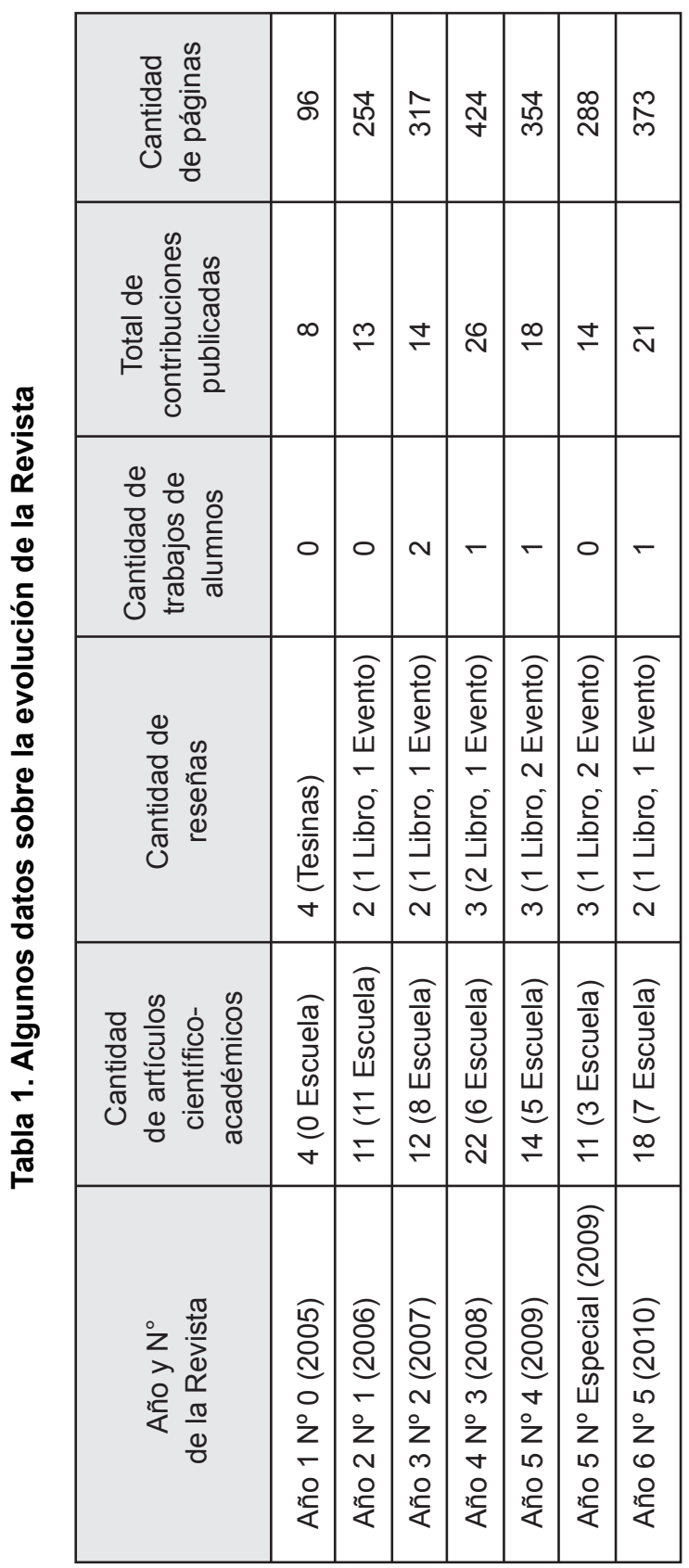


Tabla 2. Instituciones de pertenencia de los autores

\begin{tabular}{|c|l|}
\hline \multicolumn{1}{|c|}{$\begin{array}{c}\text { Año y N } \\
\text { de la Revista }\end{array}$} & \multicolumn{1}{|c|}{ Instituciones de pertenencia de los autores } \\
\hline Año 1 No 0 (2005) & $\begin{array}{l}\text { Universidad de Santiago de Compostela - España } \\
\text { Universidad de Barcelona - España } \\
\text { Universidad Metodista de Piracicaba - Brasil } \\
\text { Instituto Superior de Ciencias de la Educación - México }\end{array}$ \\
\hline Año 2 No 1 (2006) & Universidad Nacional de Rosario \\
\hline Año 3 No 2 (2007) & $\begin{array}{l}\text { Universidad Nacional de Rosario } \\
\text { Universidad Nacional de La Plata } \\
\text { Universidad Nacional de General Sarmiento } \\
\text { Universidad de Buenos Aires } \\
\text { Consejo Nacional de Investigaciones Científicas y Técnicas }\end{array}$ \\
\hline Año 4 No 3 (2008) & $\begin{array}{l}\text { Universidad Nacional de Rosario } \\
\text { Universidad Nacional de Entre Ríos } \\
\text { Universidad Nacional de La Plata } \\
\text { Universidad Nacional del Litoral } \\
\text { Universidad de Buenos Aires } \\
\text { Universidad Autónoma de Entre Ríos } \\
\text { Universidad Tecnológica Nacional } \\
\text { Universidad Abierta Interamericana } \\
\text { Universidad Católica de Córdoba } \\
\text { Facultad Latinoamericana de Ciencias Sociales } \\
\text { Instituto Rosario de Investigaciones en Ciencias } \\
\text { de la Educación } \\
\text { Instituto para la Inclusión Social y el Desarrollo Humano } \\
\text { Consejo Nacional de Investigaciones Científicas y Técnicas } \\
\text { Universidad de Salzburg - Austria } \\
\text { Universidad de Granada - España } \\
\text { Universidad Pedagógica Nacional - Colombia } \\
\text { Universidad Autónoma de Guerrero - México } \\
\text { Instituto Politécnico Nacional - México }\end{array}$ \\
\hline
\end{tabular}


(viene de página 308)

Tabla 2. Instituciones de pertenencia de los autores

\begin{tabular}{|c|c|}
\hline $\begin{array}{c}\text { Año y N }{ }^{\circ} \\
\text { de la Revista }\end{array}$ & Instituciones de pertenencia de los autores \\
\hline Año $5 \mathrm{~N}^{\circ} 4$ (2009) & $\begin{array}{l}\text { Universidad Nacional de Rosario } \\
\text { Universidad Nacional de La Plata } \\
\text { Universidad Nacional de La Pampa } \\
\text { Universidad Nacional del Comahue } \\
\text { Universidad de Buenos Aires } \\
\text { Instituto de Formación Docente Continua de San Luis } \\
\text { Instituto Superior de Formación Docente de Chubut } \\
\text { Consejo Nacional de Investigaciones Científicas y Técnicas } \\
\text { Centro de Estudios e Investigación en Ciencias Sociales }\end{array}$ \\
\hline $\begin{array}{l}\text { Año } 5 N^{\circ} \text { Especial } \\
(2009)\end{array}$ & $\begin{array}{l}\text { Universidad Nacional de Rosario } \\
\text { Universidad Nacional de Córdoba } \\
\text { Universidad Nacional de La Pampa } \\
\text { Universidad Nacional de la Patagonia Austral } \\
\text { Universidad de Buenos Aires } \\
\text { Universidad Tecnológica Nacional } \\
\text { Universidad Abierta Interamericana } \\
\text { Instituto Superior de Bellas Artes La Pampa } \\
\text { Consejo Nacional de Investigaciones Científicas y } \\
\text { Técnicas Universidad Nacional de Costa Rica - Costa Rica } \\
\text { Universidad de La Habana - Cuba } \\
\text { Instituto Tecnológico - México }\end{array}$ \\
\hline Año 6 Nº $^{\circ}(2010)$ & $\begin{array}{l}\text { Universidad Nacional de Rosario } \\
\text { Universidad Nacional de Córdoba } \\
\text { Universidad Nacional de La Plata } \\
\text { Universidad Nacional del Comahue } \\
\text { Universidad Nacional del Centro de la Provincia de } \\
\text { Buenos Aires } \\
\text { Universidad de Buenos Aires } \\
\text { Consejo Nacional de Investigaciones Científicas y } \\
\text { Técnicas Universidad de Sucre - Colombia } \\
\text { Universidad Federal Río Grande del Norte - Brasil } \\
\text { Instituto Politécnico Nacional - México }\end{array}$ \\
\hline
\end{tabular}

